\title{
Fuzzy Inventory Model for Deteriorating Items with Time-varying Demand and Shortages
}

\author{
Chandra K. Jaggi ${ }^{1, *}$, Sarla Pareek ${ }^{2}$, Anuj Sharma ${ }^{1}$, Nidhi ${ }^{2}$ \\ ${ }^{1}$ Department of Operational Research, Faculty of Mathematical Sciences, University of Delhi, Delhi, 110007, India \\ ${ }^{2}$ Centre for Mathematical Sciences, Ban asthali University, Banasthali, 304022, Rajasthan, India
}

\begin{abstract}
Fuzzy set theory is primarily concerned with how to quantitatively deal with imp rec ision and uncertainty, and offers the decision maker another tool in addition to the classical deterministic and probabilistic mathematical tools that a re used in modeling real-world problems. The present study investigates a fuzzy economic order quantity model for deteriorating items in which demand increases with time. Shortages are allowed and fully backlogged. The demand, holding cost, unit cost, shortage cost and deterioration rate are taken as a triangular fuzzy numbers. Graded Mean Representation, Signed Distance and Centroid methods are used to defuzzify the total cost function and the results obtained by these methods are compared with the help of a numerical example. Sensitivity analysis is also carried out to explore the effect of changes in the values of some of the system parameters. The proposed methodology is applicable to other inventory models under uncertainty.
\end{abstract}

Keywords Inventory, Deterio ration, Shortages, Fuzzy Variable, Triangular Fuzzy Number, Graded mean representation method, Signed distance method, Centroid method

\section{Introduction}

In conventional inventory models, uncertainties are treated as randomness and are being handled by applying the probability theory. However, in certain situations uncertainties are due to fuzziness, and such cases are dilated in the fuzzy set theory which was demonstrated by Zadeh in[12]. Kauffmann and Gupta[1] provided an introduction to fuzzy arithmetic operation and Zimmermann[4] discussed the concept of the fuzzy set theory and its applications.

Considering the fuzzy set theory in inventory modeling renders an authenticity to the model formulated since fuzziness is the closest possible approach to reality. As reality is imp recise and can only be appro ximated to a certain extent, same way, fuzzy theory helps one to incorporate uncertainties in the formu lation of the model, thus bringing it closer to reality.

Park[10] applied the fuzzy set concepts to EOQ formula by representing the inventory carrying cost with a fuzzy number and solved the economic order quantity model using fuzzy number operations based on the extension principle. Vujosevic et al.[15] used trapezoidal fuzzy number to fuzzify the order cost in the total cost of the inventory model without backorder, and got fuzzy total cost. Yao and Lee [7] introduced a backorder inventory model with fuzzy order

* Corresponding author:

ckjaggi@yahoo.com (Chandra K. Jaggi)

Published online at http://journal.sapub.org/ajor

Copyright @ 2012 Scientific \& Academic Publishing. All Rights Reserved quantity as triangular and trapezoidal fuzzy numbers and shortage cost as a crisp parameter. Gen et al.[14] expressed their input data as fuzzy nu mbers, and then the interval mean value concept was introduced to solve the inventory problem. Chang et al.[20] considered the backorder inventory problem with fuzzy backorder such that the backorder quantity is a triangular fuzzy nu mber.

Chang[21] discussed the fuzzy production inventory model for fuzzify the product quantity as triangular fuzzy number. Lee and Yao[5] proposed the inventory without backorder models in the fuzzy sense, where the order quantity is fuzzified as the triangular fuzzy number. Yao et al.[9] as sumed to be the order quantity and the total demand rate as triangular fuzzy numbers and obtained the fuzzy inventory model without shortages. Wu and Yao[11] fuzzified the order quantity and shortage quantity into triangular fuzzy numbers in an inventory model with backorder and they obtained the me mbership function of the fuzzy cost and its centroid. Yao and Chiang[8] considered the total cost of inventory without backorder. They fuzzified the total demand and cost of storing one unit per day into triangular fuzzy numbers and defuzzify by the centroid and the signed distance methods. Dutta et al.[17] developed a model in presence of fuzzy random variable demand where the optimum is achieved using a graded mean integration representation. Chang et al.[3] developed the mixture inventory model involving variable lead-time with backorders and lost sales. First they fuzzify the random lead-time demand to be a fuzzy random variable and then fuzzify the total demand to be the triangular fuzzy number 
and derive the fuzzy total cost. By the centroid method of defuzzification, they estimate the total cost in the fuzzy sense. Wee et al.[6] developed an optimal inventory model for items with imperfect quality and shortage backordering. Lin[23] developed the inventory problem for a periodic review model with variable lead-time and fuzzified the expected demand shortage and backorder rate using signed distance method to defuzzify. Roy and Samanta[2] discussed a fuzzy continuous review inventory model without backorder for deteriorating items in which the cycle time is taken as a symmetric fuzzy number. They used the signed distance method to fuzzify the total cost. Gani and Maheswari[16] developed an EOQ model with imperfect quality items with shortages where defective rate, demand, holding cost, ordering cost and shortage cost are taken as triangular fuzzy numbers. Graded mean integration method is used for defuzzification of the total profit. A meli et al. [13] developed a new inventory model to determine ordering policy for imperfect items with fuzzy defective percentage under fuzzy discounting and inflationary conditions. They used the signed distance method of defuzzification to estimate the value of total profit. Nezhad et al.[22] developed a periodic review model and a continuous review inventory model with fuzzy setup cost, holding cost and shortage cost. Also they considered the lead-time demand and the lead-time plus one period's demand as random variables. They use two methods in the name of signed distance and possibility mean value to defuzzify. Uthayakumar and Valliathl[19] developed an economic production model for Weibull deteriorating items over an infinite horizon under fuzzy environment and considered some cost component as triangular fu zzy numbers and using the signed distance method to defuzzify the cost function.

In this paper, an inventory model for deteriorating items with shortages is considered where demand, holding cost, unit cost, shortage cost and deterioration rate are assumed as a triangular fuzzy numbers. For defuzzification of the total cost function, Graded Mean Representation, Signed Distance and Centroid methods are used. By comparing the results obtained by these methods, we get the better one as an estimate of the total cost in the fuzzy sense.

\section{Preliminaries}

In order to treat fuzzy inventory model by using graded mean representation, signed distance and centroid to defuzzify, we need the following definitions.

Definition 2.1 (By Pu and Liu[18, Definition 2.1]). A fuzzy set $\tilde{a}$ on $R=(-\infty, \infty)$ is called a fuzzy point if its membership function is

$$
\mu_{a}(x)=\left\{\begin{array}{l}
1, x=a \\
0, x \neq a
\end{array}\right\}
$$

where the point a is called the support of fuzzy set $\tilde{a}$.
Definition 2.2 A fuzzy set $\left[a_{\alpha}, b_{\alpha}\right]$ where $0 \leq \alpha \leq 1$ and $a<b$ defined on $R$, is called a level of a fuzzy interval if its membership function is

$$
\mu_{\left[a_{\alpha}, b_{\alpha}\right]}(x)=\left\{\begin{array}{l}
\alpha, a \leq x \leq b \\
0, \text { otherwise }
\end{array}\right\}
$$

Definition 2.3 A fuzzy number $\tilde{A}=(a, b, c)$ where a < $\mathrm{b}<\mathrm{c}$ and defined on $R$, is called a triangular fu zzy nu mber if its membership function is

$$
\mu_{A}=\left\{\begin{array}{l}
\frac{x-a}{b-a}, a \leq x \leq b \\
\frac{c-x}{c-b}, b \leq x \leq c \\
0, \text { Otherwise }
\end{array}\right.
$$

When $a=b=c$, we have fuzzy point $(c, c, c)=\tilde{c}$.

The family of all triangular fuzzy numbers on $R$ is denoted as

$$
F_{N}=\{(a, b, c) \mid a<b<c \forall a, b, c \in R\} .
$$

The $\alpha$-cut of $\tilde{A}=(a, b, c) \in F_{N}, 0 \leq \alpha \leq 1$, is $A(\alpha)=\left[A_{L}(\alpha), A_{R}(\alpha)\right]$.

Where

$$
A_{L}(\alpha)=a+(b-a) \alpha
$$
and $A_{R}(\alpha)=c-(c-b) \alpha$ are the left and right endpoints of $A(\alpha)$.

Definition 2.4 If $\tilde{A}=(a, b, c)$ is a triangular fuzzy number then the graded mean integration representation of $\tilde{A}$ is defined as

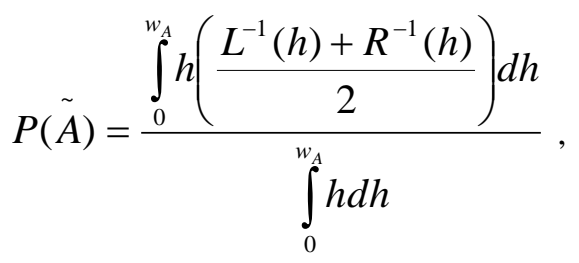

with $0<\mathrm{h} \leq w_{A}$ and $0<w_{A} \leq 1$.

$$
\begin{aligned}
& P(\tilde{A})=1 / 2 \frac{\int_{0}^{1} h[a+h(b-a)+c-h(c-a)] d h}{\int_{0}^{1} h d h} . \\
& =\frac{a+4 b+c}{6}
\end{aligned}
$$

Definition 2.5 If $\tilde{A}=(a, b, c)$ is a triangular fuzzy 
number then the signed distance of $\tilde{A}$ is defined as

$$
d(\tilde{A}, \tilde{0})=\int_{0}^{1} d\left(\left[A_{L}(\alpha)_{\alpha}, A_{R}(\alpha)_{\alpha}\right], \tilde{0}\right)=\frac{1}{4}(a+2 b+c) \text { (5) }
$$

Definition 2.6 The centroid method on the triangular fuzzy number $\tilde{A}=(a, b, c)$ is defined as

$$
C(\tilde{A})=\frac{a+b+c}{3} \text {. }
$$

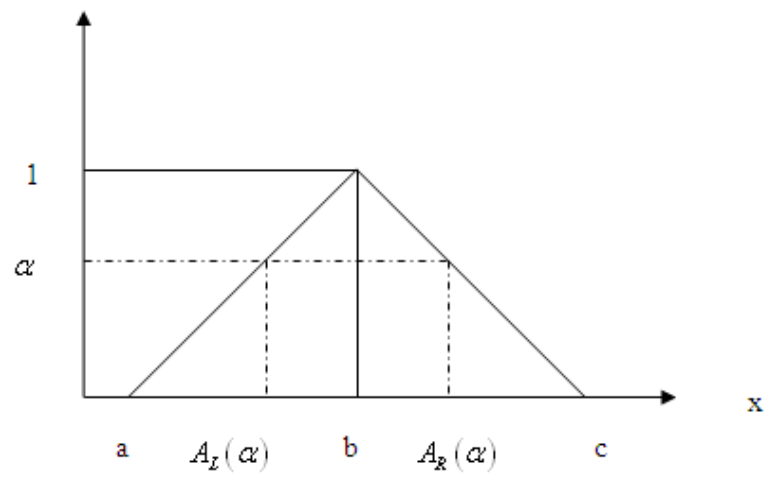

Figure. $\alpha$-cut of atriangular fuzzy number

\section{Assumptions and Notations}

The mathematical model in this paper is developed on the basis of the following assumptions and notations.

\subsection{Notations}

(i) $\rightarrow D(t)$ is the demand rate at any time $t$ per unit time.

(ii) $\rightarrow A$ is the ordering cost per order.

(iii) $\rightarrow \theta$ is the deterioration rate, $0<\theta<<1$

(iv) $\rightarrow T$ is the length of the Cycle.

(v) $\rightarrow Q$ is the ordering Quantity per unit.

(vi) $\rightarrow h$ is the holding cost per unit per unit time.

(vii) $\rightarrow S$ is the shortage Cost per unit time.

(viii) $\rightarrow C$ is the unit Cost per unit time.

(ix) $\rightarrow K\left(t_{1}, T\right)$ is the total inventory cost per unit time.

$(\mathrm{x}) \rightarrow \tilde{D}$ is the fuzzy demand.

(xi) $\rightarrow \tilde{\theta}$ is the fuzzy deterio ration rate.

(xii) $\rightarrow \tilde{h}$ is the fuzzy holding cost per unit per unit time. (xiii) $\rightarrow \tilde{S}$ is the fuzzy shortage Cost per unit time.

(xiv) $\rightarrow \tilde{C}$ is the fuzzy unit Cost per unit time.

$(\mathrm{xv}) \rightarrow \tilde{K}\left(t_{1}, T\right)$ is the total fuzzy inventory cost per unit time.

$($ xvi $) \rightarrow K_{d G}\left(t_{1}, T\right)$ is the defuzzify value of $\tilde{K}\left(t_{1}, T\right)$ by applying Graded mean integration method

(xvii) $\rightarrow K_{d S}\left(t_{1}, T\right)$ is the defuzzify value of $\tilde{K}\left(t_{1}, T\right)$ by applying Signed distance method

$\left(\right.$ xviii) $\rightarrow K_{d C}\left(t_{1}, T\right)$ is the defuzzify value of $\tilde{K}\left(t_{1}, T\right)$ by applying Centroid method.

\subsection{Assumptions}

(i) $\rightarrow$ Demand $D(t)=a(1+b t)$ is assumed to be an increasing function of time i.e. where $a$ and $b$ are positive constants and $a>0,0<b<1$.

(ii) $\rightarrow$ Replenishment is instantaneous and lead-time is zero.

(iii) $\rightarrow$ Shortages are allowed and fully backlogged.

\section{Mathematical Model}

Let $I(t)$ be the on-hand inventory at time $\mathrm{t}$ with initial inventory $Q$. During the period $\left[0, t_{1}\right]$ the on-hand inventory depletes due to demand and deterioration and exhausted at time $t_{1}$. The period $\left[t_{1}, \mathrm{~T}\right]$ is the period of shortages, which are fully backlogged. At any instant of time, the inventory level $I(t)$ is governed by the differential equations.

\subsection{Crisp Model}

$$
\frac{d I(t)}{d t}+\theta I(t)=-D(t)
$$$$
0 \leq t \leq t_{1}
$$

With $I(0)=Q$ and $I\left(t_{1}\right)=0$.

$$
\frac{d I(t)}{d t}=-D(t)
$$$$
t_{1} \leq t \leq T
$$

With $I\left(t_{1}\right)=0$.

The solution of equation (4.1) and (4.2) is given by

$$
I(t)=Q e^{-\theta t}+\left(\frac{a}{\theta}-\frac{a b}{\theta^{2}}\right) e^{-\theta t}+\frac{a b}{\theta^{2}}-\frac{a}{\theta}(1+b t)
$$

and

$$
I(t)=a\left(t_{1}-t\right)+\frac{a b}{2}\left(t_{1}^{2}-t^{2}\right)
$$


By using $I\left(t_{1}\right)=0$, we have

$$
Q=\left\{\frac{a}{\theta}\left(1+b t_{1}\right)-\frac{a b}{\theta^{2}}\right\} e^{\theta t_{1}-}\left(\frac{a}{\theta}-\frac{a b}{\theta^{2}}\right)
$$

Now, (4.3) becomes

$$
I(t)=a\left\{\left(t_{1}-t\right)+\frac{\theta}{2}\left(t_{1}-t\right)^{2}\right\}+a b\left\{t_{1}\left(t_{1}-t\right)-\frac{\left(t_{1}-t\right)^{2}}{2}+\frac{\theta}{2} t_{1}\left(t_{1}-t\right)^{2}-\frac{\theta}{6}\left(t_{1}-t\right)^{3}\right\}
$$

(Neglecting higher powers of $\theta$ ).

Total average no. of holding units $\left(I_{H}\right)$ during period $[0, \mathrm{~T}]$ is given by

$$
I_{H}=\int_{0}^{t_{1}} I(t) d t=a\left\{\frac{t_{1}^{2}}{2}+\frac{\theta}{6} t_{1}^{3}\right\}+a b\left\{\frac{t_{1}^{3}}{3}+\frac{\theta}{8} t_{1}^{4}\right\}
$$

Total no. of deteriorated units $\left(I_{D}\right)$ during period $[0, \mathrm{~T}]$ is given by

$I_{D}=Q-$ Total Demand

$$
I_{D}=Q-\int_{0}^{t_{1}} a(1+b t) d t=\frac{1}{2} a \theta t_{1}^{2}+\frac{1}{3} a b \theta t_{1}^{3}
$$

Total average no. of shortage units $\left(I_{S}\right)$ during period $[0, \mathrm{~T}]$ is given by

$$
I_{S}=-\int_{t_{1}}^{T} I(t) d t=\frac{a}{2}\left(t_{1}-T\right)^{2}-\frac{a b}{2}\left(t_{1}^{2} T-\frac{T^{3}}{3}-\frac{2}{3} t_{1}^{3}\right)
$$

Total cost of the system per unit time is given by

$$
K\left(t_{1}, T\right)=\frac{1}{T}\left[\begin{array}{c}
K\left(t_{1}, T\right)=\frac{1}{T}\left[A+h I_{H}+C I_{D}+S I_{S}\right] \\
S\left\{\frac{a}{2}\left(t_{1}-T\right)^{2}-\frac{a b}{2}\left(t_{1}^{2} T-\frac{\theta}{6} t_{1}^{3}\right)+h a b\left(\frac{t_{1}^{3}}{3}+\frac{\theta}{8} t_{1}^{4}\right)+C\left(\frac{1}{2} t_{1}^{3}\right)\right\}
\end{array}\right]
$$

\subsection{Fuzzy Model}

Due to uncertainly in the environment it is not easy to define all the parameters precisely, accordingly we assume some of these parameters namely $\tilde{a}, \tilde{b}, \tilde{C}, \tilde{S}, \tilde{\theta}, \tilde{h}$ may change within some limits.

Let $\tilde{a}=\left(a_{1}, a_{2}, a_{3}\right), \tilde{b}=\left(b_{1}, b_{2}, b_{3}\right), \tilde{C}=\left(C_{1}, C_{2}, C_{3}\right), \tilde{S}=\left(S_{1}, S_{2}, S_{3}\right), \tilde{\theta}=\left(\theta_{1}, \theta_{2}, \theta_{3}\right), \tilde{h}=\left(h_{1}, h_{2}, h_{3}\right)$ are as triangular fuzzy nu mbers.

Total cost of the system per unit time in fuzzy sense is given by

$$
\tilde{K}\left(t_{1}, T\right)=\frac{1}{T}\left[\begin{array}{l}
\left.A+\tilde{h} \tilde{a}\left(\frac{t_{1}^{2}}{2}+\frac{\theta}{6} t_{1}^{3}\right)+\tilde{h} \tilde{a} \tilde{b}\left(\frac{t_{1}^{3}}{3}+\frac{\theta}{8} t_{1}^{4}\right)+\tilde{C}\left(\frac{1}{2} \tilde{a} \tilde{\theta} t_{1}^{2}+\frac{1}{3} \tilde{a} \tilde{b} \tilde{\theta} t_{1}^{3}\right)+\right] \\
\tilde{S}\left\{\frac{\tilde{a}}{2}\left(t_{1}-T\right)^{2}-\frac{\tilde{a} \tilde{b}}{2}\left(t_{1}^{2} T-\frac{T^{3}}{3}-\frac{2}{3} t_{1}^{3}\right)\right\}
\end{array}\right]
$$

We defuzzify the fuzzy total cost $\tilde{K}\left(t_{1}, T\right)$ by graded mean representation, signed distance and centroid methods.

(i) By Graded Mean Representation Method, Total Cost is given by 
Where

$$
K_{d G}\left(t_{1}, T\right)=\frac{1}{6}\left[K_{d G_{1}}\left(t_{1}, T\right), K_{d G_{2}}\left(t_{1}, T\right), K_{d G_{3}}\left(t_{1}, T\right)\right]
$$

$$
\begin{aligned}
& K_{d G_{i}}\left(t_{1}, T\right)=\frac{1}{T}\left[\begin{array}{l}
\left.A+h_{1} a_{1}\left(\frac{t_{1}^{2}}{2}+\frac{\theta_{1}}{6} t_{1}^{3}\right)+h_{1} a_{1} b_{1}\left(\frac{t_{1}^{3}}{3}+\frac{\theta_{1}}{8} t_{1}^{4}\right)+C_{1}\left(\frac{1}{2} a_{1} \theta_{1} t_{1}^{2}+\frac{1}{3} a_{1} b_{1} \theta_{1} t_{1}^{3}\right)+\right] \\
S_{1}\left\{\frac{a_{1}}{2}\left(t_{1}-T\right)^{2}+\frac{a_{1} b_{1}}{2}\left(\frac{T^{3}}{3}+\frac{2}{3} t_{1}^{3}\right)\right\}-\frac{S_{3} a_{3} b_{3}}{2}\left(t_{1}^{2} T\right)
\end{array}\right] \\
& K_{d G_{2}}\left(t_{1}, T\right)=\frac{1}{T}\left[\begin{array}{l}
\left.A+h_{2} a_{2}\left(\frac{t_{1}^{2}}{2}+\frac{\theta_{2}}{6} t_{1}^{3}\right)+h_{2} a_{2} b_{2}\left(\frac{t_{1}^{3}}{3}+\frac{\theta_{2}}{8} t_{1}^{4}\right)+C_{2}\left(\frac{1}{2} a_{2} \theta_{2} t_{1}^{2}+\frac{1}{3} a_{2} b_{2} \theta_{2} t_{1}^{3}\right)+\right] \\
S_{2}\left\{\frac{a_{2}}{2}\left(t_{1}-T\right)^{2}-\frac{a_{2} b_{2}}{2}\left(t_{1}^{2} T-\frac{T^{3}}{3}-\frac{2}{3} t_{1}^{3}\right)\right\}
\end{array}\right] \\
& K_{d G_{3}}\left(t_{1}, T\right)=\frac{1}{T}\left[\begin{array}{l}
\left.A+h_{3} a_{3}\left(\frac{t_{1}^{2}}{2}+\frac{\theta_{3}}{6} t_{1}^{3}\right)+h_{3} a_{3} b_{3}\left(\frac{t_{1}^{3}}{3}+\frac{\theta_{3}}{8} t_{1}^{4}\right)+C_{3}\left(\frac{1}{2} a_{3} \theta_{3} t_{1}^{2}+\frac{1}{3} a_{3} b_{3} \theta_{3} t_{1}^{3}\right)+\right] \\
S_{3}\left\{\frac{a_{3}}{2}\left(t_{1}-T\right)^{2}+\frac{a_{3} b_{3}}{2}\left(\frac{T^{3}}{3}+\frac{2}{3} t_{1}^{3}\right)\right\}-\frac{S_{1} a_{1} b_{1}}{2}\left(t_{1}^{2} T\right)
\end{array}\right] \\
& K_{d G}\left(t_{1}, T\right)=\frac{1}{6}\left[K_{d G_{1}}\left(t_{1}, T\right)+4 K_{d G_{2}}\left(t_{1}, T\right)+K_{d G_{3}}\left(t_{1}, T\right)\right]
\end{aligned}
$$

To minimize total cost function per unit time $K_{d G}\left(t_{1}, T\right)$, the optimal value of $t_{1}$ and $T$ can be obtained by solving the following equations:

$$
\frac{\partial K_{d G}\left(t_{1}, T\right)}{\partial t_{1}}=0 \text { and } \frac{\partial K_{d G}\left(t_{1}, T\right)}{\partial T}=0
$$

Equation (4.13) is equivalent to

$$
\left.\frac{1}{6 T}\left[\begin{array}{l}
h_{1} a_{1}\left(t_{1}+\frac{\theta_{1}}{2} t_{1}^{2}\right)+h_{1} a_{1} b_{1}\left(t_{1}^{2}+\frac{\theta_{1}}{2} t_{1}^{3}\right)+C_{1}\left(a_{1} \theta_{1} t_{1}+a_{1} b_{1} \theta_{1} t_{1}^{2}\right)+ \\
S_{1}\left\{a_{1}\left(t_{1}-T\right)+a_{1} b_{1} t_{1}^{2}\right\}-S_{3} a_{3} b_{3} t_{1} T+ \\
h_{2} a_{2}\left(t_{1}+\frac{\theta_{2}}{2} t_{1}^{2}\right)+h_{2} a_{2} b_{2}\left(t_{1}^{2}+\frac{\theta_{2}}{2} t_{1}^{3}\right)+C_{2}\left(a_{2} \theta_{2} t_{1}+a_{2} b_{2} \theta_{2} t_{1}^{2}\right)+ \\
S_{2}\left\{a_{2}\left(t_{1}-T\right)-a_{2} b_{2}\left(t_{1} T-t_{1}^{2}\right)\right\} \\
h_{3} a_{3}\left(t_{1}+\frac{\theta_{3}}{2} t_{1}^{2}\right)+h_{3} a_{3} b_{3}\left(t_{1}^{2}+\frac{\theta_{3}}{2} t_{1}^{3}\right)+C_{3}\left(a_{3} \theta_{3} t_{1}+a_{3} b_{3} \theta_{3} t_{1}^{2}\right)+ \\
S_{3}\left\{a_{3}\left(t_{1}-T\right)+a_{3} b_{3} t_{1}^{2}\right\}-S_{1} a_{1} b_{1} t_{1} T
\end{array}\right\}+\right]=0
$$

and 


$$
\left[\begin{array}{l}
\frac{1}{6 T}\left\{\begin{array}{l}
S_{1}\left\{-a_{1}\left(t_{1}-T\right)+\frac{1}{2} a_{1} b_{1} T^{2}\right\}-\frac{1}{2} S_{3} a_{3} b_{3} t_{1}^{2}+4 S_{2}\left\{-a_{2}\left(t_{1}-T\right)-\frac{1}{2} a_{2} b_{2}\left(t_{1}^{2}-T^{2}\right)\right\} \\
+S_{3}\left\{-a_{3}\left(t_{1}-T\right)+\frac{1}{2} a_{3} b_{3} T^{2}\right\}-\frac{1}{2} S_{1} a_{1} b_{1} t_{1}^{2}
\end{array}\right\}- \\
\left.\frac{1}{6 T^{2}}\left\{\begin{array}{l}
6 A+h_{1} a_{1}\left(\frac{t_{1}^{2}}{2}+\frac{\theta_{1}}{6} t_{1}^{3}\right)+h_{1} a_{1} b_{1}\left(\frac{t_{1}^{3}}{3}+\frac{\theta_{1}}{8} t_{1}^{4}\right)+C_{1}\left(\frac{1}{2} a_{1} \theta_{1} t_{1}^{2}+\frac{1}{3} a_{1} b_{1} \theta_{1} t_{1}^{3}\right)+ \\
S_{1}\left\{\frac{a_{1}}{2}\left(t_{1}-T\right)^{2}+\frac{a_{1} b_{1}}{2}\left(\frac{T^{3}}{3}+\frac{2}{3} t_{1}^{3}\right)\right\}-\frac{S_{3} a_{3} b_{3}}{2}\left(t_{1}^{2} T\right)+4 \\
h_{2} a_{2}\left(\frac{t_{1}^{2}}{2}+\frac{\theta_{2}}{6} t_{1}^{3}\right)+h_{2} a_{2} b_{2}\left(\frac{t_{1}^{3}}{3}+\frac{\theta_{2}}{8} t_{1}^{4}\right)+C_{2}\left(\frac{1}{2} a_{2} \theta_{2} t_{1}^{2}+\frac{1}{3} a_{2} b_{2} \theta_{2} t_{1}^{3}\right)+ \\
S_{2}\left\{\frac{a_{2}}{2}\left(t_{1}-T\right)^{2}-\frac{a_{2} b_{2}}{2}\left(t_{1}^{2} T-\frac{T^{3}}{3}-\frac{2}{3} t_{1}^{3}\right)\right\} \\
h_{3} a_{3}\left(\frac{t_{1}^{2}}{2}+\frac{\theta_{3}}{6} t_{1}^{3}\right)+h_{3} a_{3} b_{3}\left(\frac{t_{1}^{3}}{3}+\frac{\theta_{3}}{8} t_{1}^{4}\right)+C_{3}\left(\frac{1}{2} a_{3} \theta_{3} t_{1}^{2}+\frac{1}{3} a_{3} b_{3} \theta_{3} t_{1}^{3}\right)+ \\
S_{3}\left\{\frac{a_{3}}{2}\left(t_{1}-T\right)^{2}+\frac{a_{3} b_{3}}{2}\left(\frac{T^{3}}{3}+\frac{2}{3} t_{1}^{3}\right)\right\}-\frac{S_{1} a_{1} b_{1}}{2}\left(t_{1}^{2} T\right)
\end{array}\right\}+\right\}
\end{array}\right\}
$$

Further, for the total cost function $K_{d G}\left(t_{1}, T\right)$ to be convex, the following conditions must be satisfied

$$
\begin{gathered}
\frac{\partial^{2} K_{d G}\left(t_{1}, T\right)}{\partial t_{1}^{2}}>0, \frac{\partial^{2} K_{d G}\left(t_{1}, T\right)}{\partial T^{2}}>0 \\
\text { and }\left(\frac{\partial^{2} K_{d G}\left(t_{1}, T\right)}{\partial t_{1}^{2}}\right)\left(\frac{\partial^{2} K_{d G}\left(t_{1}, T\right)}{\partial T^{2}}\right)-\left(\frac{\partial^{2} K_{d G}\left(t_{1}, T\right)}{\partial t_{1} \partial T}\right)>0 .
\end{gathered}
$$

The second derivatives of the total cost function $K_{d G}\left(t_{1}, T\right)$ are complicated and it is very difficult to prove the convexity mathematically. Thus, the convexity of total cost function has been established graphically, (Figure (A)).

(ii) By Signed Distance Method, Total cost is given by

$$
K_{d S}\left(t_{1}, T\right)=\frac{1}{4}\left[K_{d S_{1}}\left(t_{1}, T\right), K_{d S_{2}}\left(t_{1}, T\right), K_{d S_{3}}\left(t_{1}, T\right)\right]
$$

Where

$$
\begin{gathered}
K_{d S_{i}}\left(t_{1}, T\right)=\frac{1}{T}\left[\begin{array}{l}
\left.A+h_{1} a_{1}\left(\frac{t_{1}^{2}}{2}+\frac{\theta_{1}}{6} t_{1}^{3}\right)+h_{1} a_{1} b_{1}\left(\frac{t_{1}^{3}}{3}+\frac{\theta_{1}}{8} t_{1}^{4}\right)+C_{1}\left(\frac{1}{2} a_{1} \theta_{1} t_{1}^{2}+\frac{1}{3} a_{1} b_{1} \theta_{1} t_{1}^{3}\right)+\right] \\
S_{1}\left\{\frac{a_{1}}{2}\left(t_{1}-T\right)^{2}+\frac{a_{1} b_{1}}{2}\left(\frac{T^{3}}{3}+\frac{2}{3} t_{1}^{3}\right)\right\}-\frac{S_{3} a_{3} b_{3}}{2}\left(t_{1}^{2} T\right)
\end{array}\right] \\
K_{d S_{2}}\left(t_{1}, T\right)=\frac{1}{T}\left[\begin{array}{l}
\left.A+h_{2} a_{2}\left(\frac{t_{1}^{2}}{2}+\frac{\theta_{2}}{6} t_{1}^{3}\right)+h_{2} a_{2} b_{2}\left(\frac{t_{1}^{3}}{3}+\frac{\theta_{2}}{8} t_{1}^{4}\right)+C_{2}\left(\frac{1}{2} a_{2} \theta_{2} t_{1}^{2}+\frac{1}{3} a_{2} b_{2} \theta_{2} t_{1}^{3}\right)+\right] \\
S_{2}\left\{\frac{a_{2}}{2}\left(t_{1}-T\right)^{2}-\frac{a_{2} b_{2}}{2}\left(t_{1}^{2} T-\frac{T^{3}}{3}-\frac{2}{3} t_{1}^{3}\right)\right\}
\end{array}\right. \\
K_{d S_{3}}\left(t_{1}, T\right)=\frac{1}{T}\left[\begin{array}{l}
\left.A+h_{3} a_{3}\left(\frac{t_{1}^{2}}{2}+\frac{\theta_{3}}{6} t_{1}^{3}\right)+h_{3} a_{3} b_{3}\left(\frac{t_{1}^{3}}{3}+\frac{\theta_{3}}{8} t_{1}^{4}\right)+C_{3}\left(\frac{1}{2} a_{3} \theta_{3} t_{1}^{2}+\frac{1}{3} a_{3} b_{3} \theta_{3} t_{1}^{3}\right)+\right] \\
S_{3}\left\{\frac{a_{3}}{2}\left(t_{1}-T\right)^{2}+\frac{a_{3} b_{3}}{2}\left(\frac{T^{3}}{3}+\frac{2}{3} t_{1}^{3}\right)\right\}-\frac{S_{1} a_{1} b_{1}}{2}\left(t_{1}^{2} T\right)
\end{array}\right]
\end{gathered}
$$




$$
K_{d S}\left(t_{1}, T\right)=\frac{1}{4}\left[K_{d S_{1}}\left(t_{1}, T\right)+2 K_{d S_{2}}\left(t_{1}, T\right)+K_{d S_{3}}\left(t_{1}, T\right)\right]
$$

The total cost function $K_{d S}\left(t_{1}, T\right)$ has been minimized follo wing the same process as has been stated in case (i).

To minimize total cost function per unit time $K_{d S}\left(t_{1}, T\right)$, the optimal value of $t_{1}$ and $T$ can be obtained by solving the following equations:

$$
\frac{\partial K_{d S}\left(t_{1}, T\right)}{\partial t_{1}}=0 \text { and } \frac{\partial K_{d S}\left(t_{1}, T\right)}{\partial T}=0
$$

Equation (4.19) is equivalent to

$$
\left.\frac{1}{4 T}\left[\begin{array}{l}
h_{1} a_{1}\left(t_{1}+\frac{\theta_{1}}{2} t_{1}^{2}\right)+h_{1} a_{1} b_{1}\left(t_{1}^{2}+\frac{\theta_{1}}{2} t_{1}^{3}\right)+C_{1}\left(a_{1} \theta_{1} t_{1}+a_{1} b_{1} \theta_{1} t_{1}^{2}\right)+ \\
S_{1}\left\{a_{1}\left(t_{1}-T\right)+a_{1} b_{1} t_{1}^{2}\right\}-S_{3} a_{3} b_{3} t_{1} T+ \\
h_{2} a_{2}\left(t_{1}+\frac{\theta_{2}}{2} t_{1}^{2}\right)+h_{2} a_{2} b_{2}\left(t_{1}^{2}+\frac{\theta_{2}}{2} t_{1}^{3}\right)+C_{2}\left(a_{2} \theta_{2} t_{1}+a_{2} b_{2} \theta_{2} t_{1}^{2}\right)+ \\
S_{2}\left\{a_{2}\left(t_{1}-T\right)-a_{2} b_{2}\left(t_{1} T-t_{1}^{2}\right)\right\} \\
h_{3} a_{3}\left(t_{1}+\frac{\theta_{3}}{2} t_{1}^{2}\right)+h_{3} a_{3} b_{3}\left(t_{1}^{2}+\frac{\theta_{3}}{2} t_{1}^{3}\right)+C_{3}\left(a_{3} \theta_{3} t_{1}+a_{3} b_{3} \theta_{3} t_{1}^{2}\right)+ \\
S_{3}\left\{a_{3}\left(t_{1}-T\right)+a_{3} b_{3} t_{1}^{2}\right\}-S_{1} a_{1} b_{1} t_{1} T
\end{array}\right\}+\right]=0
$$

and

$$
\left[\begin{array}{l}
\frac{1}{4 T}\left\{\begin{array}{l}
S_{1}\left\{-a_{1}\left(t_{1}-T\right)+\frac{1}{2} a_{1} b_{1} T^{2}\right\}-\frac{1}{2} S_{3} a_{3} b_{3} t_{1}^{2}+2 S_{2}\left\{-a_{2}\left(t_{1}-T\right)-\frac{1}{2} a_{2} b_{2}\left(t_{1}^{2}-T^{2}\right)\right\} \\
+S_{3}\left\{-a_{3}\left(t_{1}-T\right)+\frac{1}{2} a_{3} b_{3} T^{2}\right\}-\frac{1}{2} S_{1} a_{1} b_{1} t_{1}^{2}
\end{array}\right\}- \\
\left.\frac{1}{4 T^{2}}\left\{\begin{array}{l}
4 A+h_{1} a_{1}\left(\frac{t_{1}^{2}}{2}+\frac{\theta_{1}}{6} t_{1}^{3}\right)+h_{1} a_{1} b_{1}\left(\frac{t_{1}^{3}}{3}+\frac{\theta_{1}}{8} t_{1}^{4}\right)+C_{1}\left(\frac{1}{2} a_{1} \theta_{1} t_{1}^{2}+\frac{1}{3} a_{1} b_{1} \theta_{1} t_{1}^{3}\right)+ \\
S_{1}\left\{\frac{a_{1}}{2}\left(t_{1}-T\right)^{2}+\frac{a_{1} b_{1}}{2}\left(\frac{T^{3}}{3}+\frac{2}{3} t_{1}^{3}\right)\right\}-\frac{S_{3} a_{3} b_{3}}{2}\left(t_{1}^{2} T\right)+2 \\
h_{2} a_{2}\left(\frac{t_{1}^{2}}{2}+\frac{\theta_{2}}{6} t_{1}^{3}\right)+h_{2} a_{2} b_{2}\left(\frac{t_{1}^{3}}{3}+\frac{\theta_{2}}{8} t_{1}^{4}\right)+C_{2}\left(\frac{1}{2} a_{2} \theta_{2} t_{1}^{2}+\frac{1}{3} a_{2} b_{2} \theta_{2} t_{1}^{3}\right)+ \\
S_{2}\left\{\frac{a_{2}}{2}\left(t_{1}-T\right)^{2}-\frac{a_{2} b_{2}}{2}\left(t_{1}^{2} T-\frac{T^{3}}{3}-\frac{2}{3} t_{1}^{3}\right)\right\} \\
h_{3} a_{3}\left(\frac{t_{1}^{2}}{2}+\frac{\theta_{3}}{6} t_{1}^{3}\right)+h_{3} a_{3} b_{3}\left(\frac{t_{1}^{3}}{3}+\frac{\theta_{3}}{8} t_{1}^{4}\right)+C_{3}\left(\frac{1}{2} a_{3} \theta_{3} t_{1}^{2}+\frac{1}{3} a_{3} b_{3} \theta_{3} t_{1}^{3}\right)+ \\
S_{3}\left\{\frac{a_{3}}{2}\left(t_{1}-T\right)^{2}+\frac{a_{3} b_{3}}{2}\left(\frac{T^{3}}{3}+\frac{2}{3} t_{1}^{3}\right)\right\}-\frac{S_{1} a_{1} b_{1}}{2}\left(t_{1}^{2} T\right)
\end{array}\right\}+\right\}
\end{array}\right\}
$$

Further, for the total cost function $K_{d S}\left(t_{1}, T\right)$ to be convex, the following conditions must be satisfied

$$
\frac{\partial^{2} K_{d S}\left(t_{1}, T\right)}{\partial t_{1}^{2}}>0, \frac{\partial^{2} K_{d S}\left(t_{1}, T\right)}{\partial T^{2}}>0
$$




$$
\text { and }\left(\frac{\partial^{2} K_{d S}\left(t_{1}, T\right)}{\partial t_{1}^{2}}\right)\left(\frac{\partial^{2} K_{d S}\left(t_{1}, T\right)}{\partial T^{2}}\right)-\left(\frac{\partial^{2} K_{d S}\left(t_{1}, T\right)}{\partial t_{1} \partial T}\right)>0 \text {. }
$$

The second derivatives of the total cost function $K_{d S}\left(t_{1}, T\right)$ are complicated and it is very difficult to prove the convexity mathematically. Thus, the convexity of total cost function has been established graphically, (Figure (B)).

(iii) By Centroid Method, Total cost is given by

Where

$$
K_{d C}\left(t_{1}, T\right)=\frac{1}{3}\left[K_{d C_{1}}\left(t_{1}, T\right), K_{d C_{2}}\left(t_{1}, T\right), K_{d C_{3}}\left(t_{1}, T\right)\right]
$$

$$
\begin{gathered}
K_{d C_{1}}\left(t_{1}, T\right)=\frac{1}{T}\left[\begin{array}{l}
\left.A+h_{1} a_{1}\left(\frac{t_{1}^{2}}{2}+\frac{\theta_{1}}{6} t_{1}^{3}\right)+h_{1} a_{1} b_{1}\left(\frac{t_{1}^{3}}{3}+\frac{\theta_{1}}{8} t_{1}^{4}\right)+C_{1}\left(\frac{1}{2} a_{1} \theta_{1} t_{1}^{2}+\frac{1}{3} a_{1} b_{1} \theta_{1} t_{1}^{3}\right)+\right] \\
S_{1}\left\{\frac{a_{1}}{2}\left(t_{1}-T\right)^{2}+\frac{a_{1} b_{1}}{2}\left(\frac{T^{3}}{3}+\frac{2}{3} t_{1}^{3}\right)\right\}-\frac{S_{3} a_{3} b_{3}}{2}\left(t_{1}^{2} T\right)
\end{array}\right] \\
K_{d C_{2}}\left(t_{1}, T\right)=\frac{1}{T}\left[\begin{array}{l}
\left.A+h_{2} a_{2}\left(\frac{t_{1}^{2}}{2}+\frac{\theta_{2}}{6} t_{1}^{3}\right)+h_{2} a_{2} b_{2}\left(\frac{t_{1}^{3}}{3}+\frac{\theta_{2}}{8} t_{1}^{4}\right)+C_{2}\left(\frac{1}{2} a_{2} \theta_{2} t_{1}^{2}+\frac{1}{3} a_{2} b_{2} \theta_{2} t_{1}^{3}\right)+\right] \\
S_{2}\left\{\frac{a_{2}}{2}\left(t_{1}-T\right)^{2}-\frac{a_{2} b_{2}}{2}\left(t_{1}^{2} T-\frac{T^{3}}{3}-\frac{2}{3} t_{1}^{3}\right)\right\} \\
K_{d C_{3}}\left(t_{1}, T\right)=\frac{1}{T}\left[\begin{array}{l}
\left.A+h_{3} a_{3}\left(\frac{t_{1}^{2}}{2}+\frac{\theta_{3}}{6} t_{1}^{3}\right)+h_{3} a_{3} b_{3}\left(\frac{t_{1}^{3}}{3}+\frac{\theta_{3}}{8} t_{1}^{4}\right)+C_{3}\left(\frac{1}{2} a_{3} \theta_{3} t_{1}^{2}+\frac{1}{3} a_{3} b_{3} \theta_{3} t_{1}^{3}\right)+\right] \\
S_{3}\left\{\frac{a_{3}}{2}\left(t_{1}-T\right)^{2}+\frac{a_{3} b_{3}}{2}\left(\frac{T^{3}}{3}+\frac{2}{3} t_{1}^{3}\right)\right\}-\frac{S_{1} a_{1} b_{1}}{2}\left(t_{1}^{2} T\right)
\end{array}\right] \\
K_{d C}\left(t_{1}, T\right)=\frac{1}{3}\left[K_{d C_{1}}\left(t_{1}, T\right)+K_{d C_{2}}\left(t_{1}, T\right)+K_{d C_{3}}\left(t_{1}, T\right)\right]
\end{array}\right]
\end{gathered}
$$

The total cost function $K_{d C}\left(t_{1}, T\right)$ has been minimized following the same process as has been stated in case (i).

To minimize total cost function per unit time $K_{d C}\left(t_{1}, T\right)$, the optimal value of $t_{1}$ and $T$ can be obtained by solving the following equations:

$$
\frac{\partial K_{d C}\left(t_{1}, T\right)}{\partial t_{1}}=0 \text { and } \frac{\partial K_{d C}\left(t_{1}, T\right)}{\partial T}=0
$$

Equation (4.25) is equivalent to

$$
\frac{1}{3 T}\left[\begin{array}{l}
h_{1} a_{1}\left(t_{1}+\frac{\theta_{1}}{2} t_{1}^{2}\right)+h_{1} a_{1} b_{1}\left(t_{1}^{2}+\frac{\theta_{1}}{2} t_{1}^{3}\right)+C_{1}\left(a_{1} \theta_{1} t_{1}+a_{1} b_{1} \theta_{1} t_{1}^{2}\right)+ \\
S_{1}\left\{a_{1}\left(t_{1}-T\right)+a_{1} b_{1} t_{1}^{2}\right\}-S_{3} a_{3} b_{3} t_{1} T+ \\
h_{2} a_{2}\left(t_{1}+\frac{\theta_{2}}{2} t_{1}^{2}\right)+h_{2} a_{2} b_{2}\left(t_{1}^{2}+\frac{\theta_{2}}{2} t_{1}^{3}\right)+C_{2}\left(a_{2} \theta_{2} t_{1}+a_{2} b_{2} \theta_{2} t_{1}^{2}\right)+ \\
S_{2}\left\{a_{2}\left(t_{1}-T\right)-a_{2} b_{2}\left(t_{1} T-t_{1}^{2}\right)\right\} \\
h_{3} a_{3}\left(t_{1}+\frac{\theta_{3}}{2} t_{1}^{2}\right)+h_{3} a_{3} b_{3}\left(t_{1}^{2}+\frac{\theta_{3}}{2} t_{1}^{3}\right)+C_{3}\left(a_{3} \theta_{3} t_{1}+a_{3} b_{3} \theta_{3} t_{1}^{2}\right)+ \\
S_{3}\left\{a_{3}\left(t_{1}-T\right)+a_{3} b_{3} t_{1}^{2}\right\}-S_{1} a_{1} b_{1} t_{1} T
\end{array}\right\}+0
$$


and

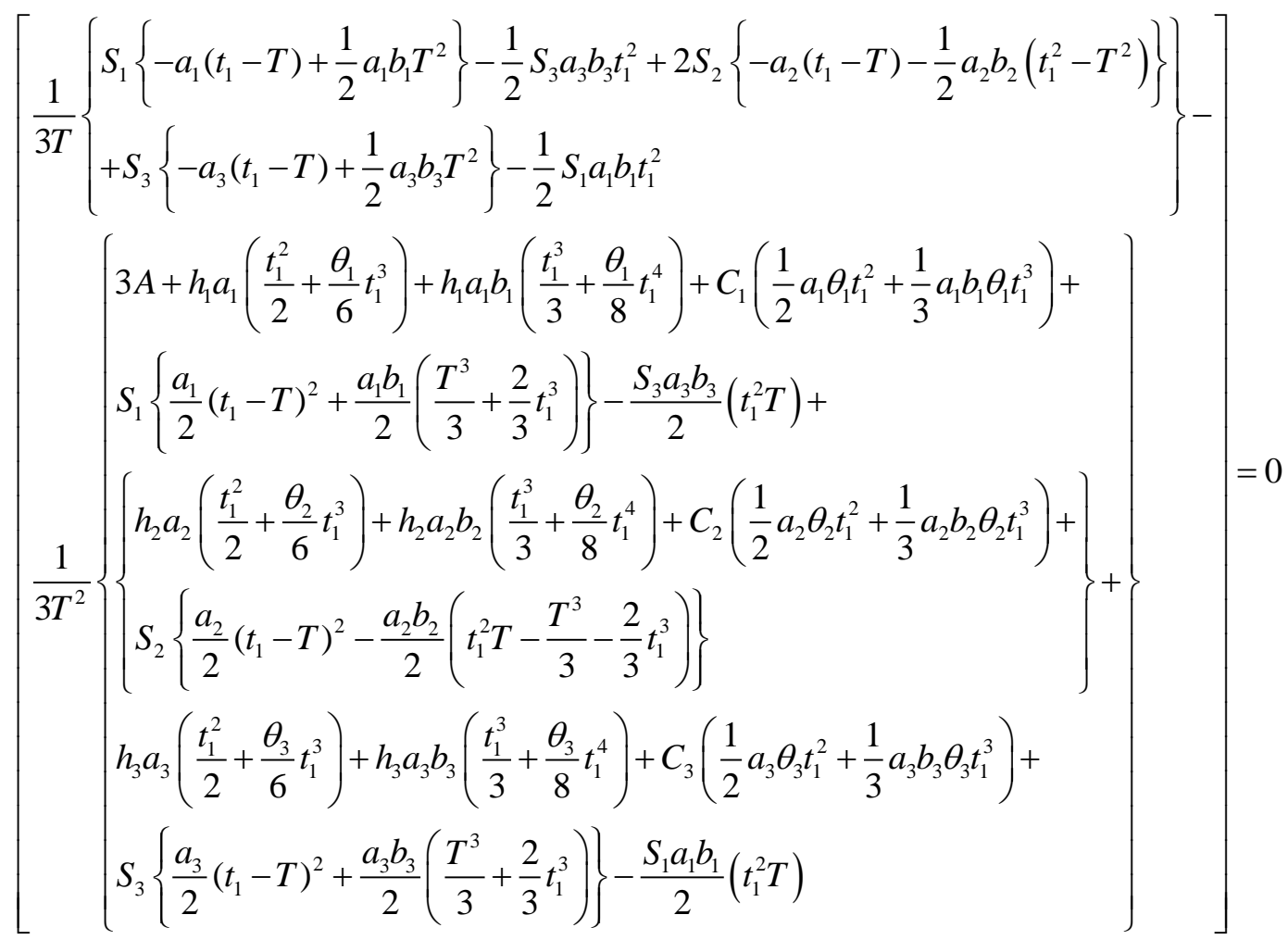

Further, for the total cost function $K_{d C}\left(t_{1}, T\right)$ to be convex, the following conditions must be satisfied

$$
\begin{gathered}
\frac{\partial^{2} K_{d C}\left(t_{1}, T\right)}{\partial t_{1}^{2}}>0, \frac{\partial^{2} K_{d C}\left(t_{1}, T\right)}{\partial T^{2}}>0 \\
\text { and }\left(\frac{\partial^{2} K_{d C}\left(t_{1}, T\right)}{\partial t_{1}^{2}}\right)\left(\frac{\partial^{2} K_{d C}\left(t_{1}, T\right)}{\partial T^{2}}\right)-\left(\frac{\partial^{2} K_{d C}\left(t_{1}, T\right)}{\partial t_{1} \partial T}\right)>0 .
\end{gathered}
$$

The second derivatives of the total cost function $K_{d C}\left(t_{1}, T\right)$ are complicated and it is very difficult to prove the convexity mathematically. Thus, the convexity of total cost function has been established graphically, (Figure (C)).

\section{Numerical Example}

Consider an inventory system with following parametric values.

Crisp Model, $A=\mathrm{Rs} 200$ /order, $C=\mathrm{Rs} 20$ /unit, $h=$ Rs. 5/unit/year, $a=100$ units/year, $b=.1$ units/year, $\theta=.01 /$ year, $S=$ Rs $15 /$ unit/year.

The solution of crisp model is

$K\left(t_{1}, T\right)=$ Rs $404.3429, t_{1}=.7149$ year, $T=.9636$ year.

Fuzzy Model,

$$
\begin{aligned}
& \tilde{a}=(60,100,140), \tilde{b}=(.06, .10, .14), \\
& \tilde{C}=(16,20,24), \tilde{S}=(12,15,18), \\
& \tilde{\theta}=(.006, .010, .014), \tilde{h}=(3,5,7)
\end{aligned}
$$

The solution of fuzzy model can be determined by following three methods.
By Graded Mean Representation Method, we have

1. When $\tilde{a}, \tilde{b}, \tilde{C}, \tilde{S}, \tilde{\theta}, \tilde{h}$ all are triangular fuzzy nu mbers $K_{d G}\left(t_{1}, T\right)=$ Rs 414.6096, $t_{1}=.6908$ year, $T=.9383$ year.

2. When $\tilde{a}, \tilde{b}, \tilde{C}, \tilde{S}, \tilde{\theta}$ are triangular fu zzy numbers $K_{d G}\left(t_{1}, T\right)=$ Rs 406.9852, $t_{1}=.7135$ year, $T=.9560$ year.

3. When $\tilde{a}, \tilde{b}, \tilde{C}, \tilde{\theta}$ are triangular fu zzy numbers

$$
K_{d G}\left(t_{1}, T\right)=\text { Rs } 405.5274, t_{1}=.7115 \text { year, } T=.9596
$$
year.

4. When $\tilde{a}, \tilde{b}$ and $\tilde{\theta}$ are triangular fuzzy numbers

$$
K_{d G}\left(t_{1}, T\right)=\text { Rs } 405.2250, t_{1}=.7120 \text { year, } T=.9603
$$
year. 
5. When $\tilde{a}$ and $\tilde{b}$ are triangular fuzzy numbers

$K_{d G}\left(t_{1}, T\right)=$ Rs 404.8978, $t_{1}=.7131$ year, $T=.9611$ year.

By Signed Distance Method, we have

1. When $\tilde{a}, \tilde{b}, \tilde{C}, \tilde{S}, \tilde{\theta}, \tilde{h}$ all are triangular fuzzy nu mbers

$K_{d S}\left(t_{1}, T\right)=\operatorname{Rs} 419.6059, t_{1}=.6797$ year, $T=.9266$ year.

2. When $\tilde{a}, \tilde{b}, \tilde{C}, \tilde{S}, \tilde{\theta}$ are triangular fuzzy numbers

$K_{d S}\left(t_{1}, T\right)=$ Rs 408.2810, $t_{1}=.7128$ year, $T=.9523$ year .

3. When $\tilde{a}, \tilde{b}, \tilde{C}, \tilde{\theta}$ are triangular fuzzy numbers

$K_{d S}\left(t_{1}, T\right)=$ Rs 406.1163, $t_{1}=.7093$ year, $T=.9576$ year.

4. When $\tilde{a}, \tilde{b}$ and $\tilde{\theta}$ are triangular fu zzy numbers

$K_{d S}\left(t_{1}, T\right)=$ Rs $405.6640, t_{1}=.7106$ year, $T=.9587$ year.

5. When $\tilde{a}$ and $\tilde{b}$ are triangular fuzzy nu mbers

$K_{d S}\left(t_{1}, T\right)=$ Rs $405.1742, t_{1}=.7122$ year, $T=.9599$ year.

By Centroid Method, we have

1. When $\tilde{a}, \tilde{b}, \tilde{C}, \tilde{S}, \tilde{\theta}, \tilde{h}$ all are triangular fuzzy nu mbers
$K_{d C}\left(t_{1}, T\right)=\operatorname{Rs} 424.5173, t_{1}=.6691$ year, $T=9153$ year.

2. When $\tilde{a}, \tilde{b}, \tilde{C}, \tilde{S}, \tilde{\theta}$ are triangular fuzzy numbers

$K_{d C}\left(t_{1}, T\right)=$ Rs $409.5606, t_{1}=.7121$ year, $T=.9487$ year.

3. When $\tilde{a}, \tilde{b}, \tilde{C}, \tilde{\theta}$ are triangular fu zzy numbers

$K_{d C}\left(t_{1}, T\right)=\operatorname{Rs} 406.7030, t_{1}=.7074$ year, $T=.9557$ year.

4. When $\tilde{a}, \tilde{b}$ and $\tilde{\theta}$ are triangular fuzzy numbers

$K_{d C}\left(t_{1}, T\right)=$ Rs 406.1016, $t_{1}=.7092$ year, $T=.9571$ year.

5. When $\tilde{a}$ and $\tilde{b}$ are triangular fuzzy nu mbers

$K_{d C}\left(t_{1}, T\right)=$ Rs $405.4499, t_{1}=.7113$ year, $T=.9587$ year.

\section{Sensitivity Analysis}

A sensitivity analys is is performed to study the effects of changes in fuzzy parameters $\tilde{a}, \tilde{b}$ and $\tilde{\theta}$ on the optimal solution by taking the defuzzify values of these parameters. The results are shown in below tables.

Table 1. Sensitivity Analysis on parameter $a$

$\begin{array}{ccrc}a \text { (units/year) } & t_{1} \text { (year) } & T \text { (year) } & K_{d G}\left(t_{1}, T\right)(\mathrm{Rs}) \\ & & & \\ 60 & .8614 & 1.1755 & 328.6584 \\ 80 & .7619 & 1.0367 & 374.1864 \\ 100 & .6908 & .9383 & 414.6096 \\ 120 & .6368 & .8638 & 451.3349 \\ 140 & .5938 & .8049 & 485.2219\end{array}$

Table1 indicates that as the value of $a$ increases, fuzzy cost $K_{d G}\left(t_{1}, T\right)$ increases significantly but $t_{1}$ and $T$ decreases drastically.

Table 2. Sensitivity Analysis on parameter $b$

$\begin{array}{cccc}b \text { (units/year) } & t_{1} \text { (year) } & T \text { (year) } & K_{d G}\left(t_{1}, T\right)(\text { Rs) } \\ & & & \\ .06 & .7041 & .9564 & 410.3971 \\ .08 & .6973 & .9472 & 412.5235 \\ .10 & .6908 & .9383 & 414.6096 \\ .12 & .6846 & .9299 & 416.6576 \\ .14 & .6787 & .9218 & 418.6696\end{array}$

Table 2 indicates that as the value of $b$ increases, fuzzy cost $K_{d G}\left(t_{1}, T\right)$ increases regularly but $t_{1}$ and $T$ decreases gradually. 
Table 3. Sensitivity Analysis on parameter $\theta$

$\begin{array}{rrrr}\theta & t_{1} \text { ( year) } & T \text { (year) } & K_{d G}\left(t_{1}, T\right)(\text { Rs) } \\ .006 & .6978 & .9437 & 412.2687 \\ .008 & .6943 & .9410 & 413.4436 \\ .010 & .6908 & .9383 & 414.6096 \\ .012 & .6874 & .9357 & 415.7667 \\ .014 & .6840 & .9331 & 416.9151\end{array}$

Table 3 indicates that as the value of $\theta$ increases, fuzzy cost $K_{d G}\left(t_{1}, T\right)$ increases slightly but $t_{1}$ and $T$ decreases gradually.

If we plot the total cost function $K_{d G}\left(t_{1}, T\right)$ with some values of $t_{1}$ and $T$ s.t. $t_{1}=.65$ to 2 with equal interval $T$ $=.84$ to 1 , then we get strictly convex graph of total cost function

$K_{d G}\left(t_{1}, T\right)$ given below.

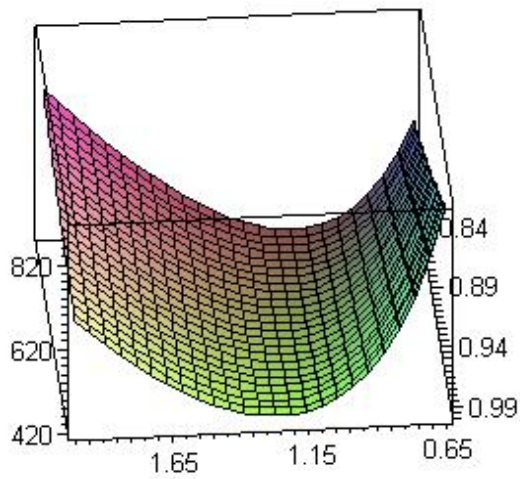

Figure (A). Total Fuzzy Cost $K_{d G}\left(t_{1}, T\right)$ vs. $t_{1}$ and $T$

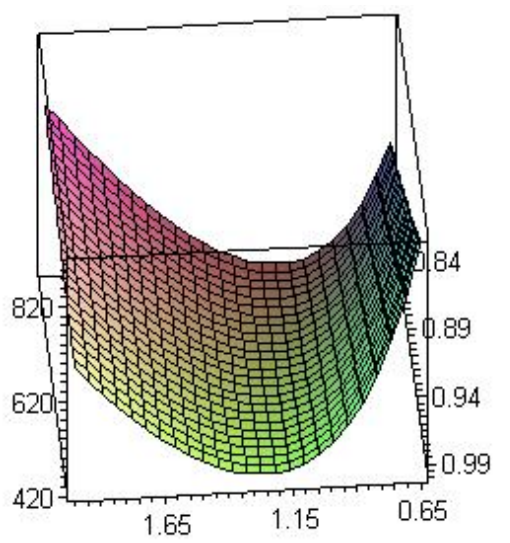

Figure (B). Total Fuzzy Cost $K_{d S}\left(t_{1}, T\right)$ Vs. $t_{1}$ and $T$

If we plot the total cost function $K_{d S}\left(t_{1}, T\right)$ with some values of $t_{1}$ and $T$ s.t. $t_{1}=.65$ to 2 with equal interval $T$ $=.84$ to 1 , then we get strictly convex graph of total cost function $K_{d S}\left(t_{1}, T\right)$ given below.

If we plot the total cost function $K_{d C}\left(t_{1}, T\right)$ with some values of $t_{1}$ and $T$ s.t. $t_{1}=.65$ to 2 with equal interval $T$ $=.84$ to 1 , then we get strictly convex graph of total cost function

$K_{d C}\left(t_{1}, T\right)$ given below.

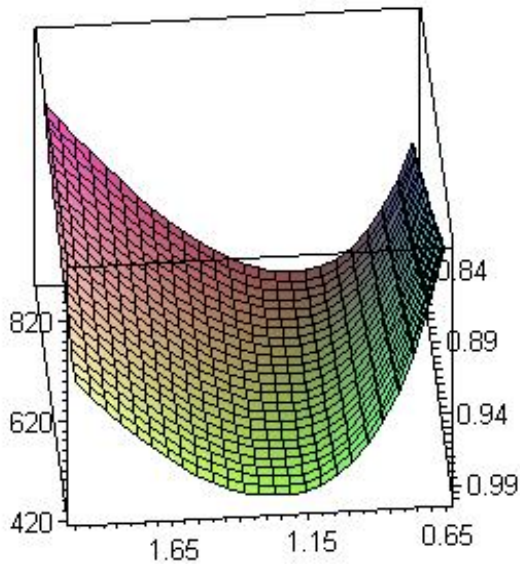

Figure (C). Total Fuzzy Cost $K_{d C}\left(t_{1}, T\right)$ vs. $t_{1}$ and $T$

\section{Conclusions}

This paper presents a fuzzy inventory model for deteriorating items with allowable shortages in which demand is an increasing function of time. The de mand, deterioration rate, inventory holding cost, unit cost and shortage cost are represented by triangular fuzzy numbers. For defuzzification, graded mean, signed distance and centroid method are employed to evaluate the optimal time period of positive stock $t_{1}$ and total cycle length $\mathrm{T}$ which minimizes the total cost. By given numerical example it has been tested that graded mean representation method gives minimu $\mathrm{m}$ cost as compared to signed distance method and centroid method. A sensitivity analysis is also conducted on the parameters $a, b$ and $\theta$ to explore the effects of fuzziness.

Finding Suggest that the change in parameters $a, b$ and $\theta$ will result the change in fuzzy cost with some changes in $t_{1}$ and $T$.With the increases values of these parameters will result in increase of fuzzy cost, but decreases $t_{1}$ and $T$. Similarly with the decreases values of these parameters will result in decrease of fuzzy cost, but increases $t_{1}$ and $T$.

A future study would be to extend the proposed model for 
finite replenishment rate, stock outs, which are partially backlogged, price dependent demand, stock dependent demand and many more.

\section{ACKNOWLEDGEMENTS}

The authors would like to thank anonymous referees for their valuable and constructive comments and suggestions that have led to improvement on the earlier version of the paper. The first author would like to acknowledge the support of the Research Grant No.: Dean(R/R\&D/2012/917), provided by the University of Delhi, Delhi, India for conducting this research. The third author would like to thank University Grants Commission for providing Junior Research Fellowship vide letter No. JRF/AA/168/2010-11, 47126 .

\section{REFERENCES}

[1] Arnold Kaufmann, Madan M Gupta, "Introduction to Fuzzy Arithmetic: Theory and Applications", Van Nostrand Reinhold, New York, 1991.

[2] Ajanta Roy, Guru P Samanta, "Fuzzy continuous review inventory model without backorder for deteriorating items", Electronic Journal of Applied Statistical Analysis, vol. 2, no.1, pp. 58-66, 2009.

[3] Hung C. Chang, Jing S Yao, Liang Y Ouyang, "Fuzzy mixture inventory model involving fuzzy random variable lead-time demand and fuzzy total demand", European Journal of Operational Research, vol. 169, no. 1, pp. 65-80, 2006.

[4] Hans J Zimmermann, "Fuzzy Set Theory and Its Applications," 3rd Ed. Dordrecht: Kluwer, Academic Publishers, 1996.

[5] Huey M Lee, Jing S Yao, "Economic order quantity in fuzzy sense for inventory without backorder model”, Fuzzy Sets and Sy stems, vol. 105, pp.13-31, 1999.

[6] Hui M Wee, Jonas Yu, Mei C Chen, "Optimal inventory model for items with imperfect quality and shortage backordering", International Journal of Management Science, vol. 35 , pp. $7-11,2007$.

[7] Jing S Yao, Huey M Lee, "Fuzzy inventory with backorder for fuzzy order quantity", Information Sciences, vol. 93, pp. 283-319, 1996.

[8] Jing S Yao, Jershan Chiang, "Inventory without backorder with fuzzy total cost and fuzzy storing cost defuzzified by centroid and signed distance", European journal of Operations research, vol. 148, pp. 401-409, 2003.

[9] Jing S Yao, San C Chang, Jin S Su, "Fuzzy Inventory without backorder for fuzzy order quantity and fuzzy total demand quantity", Computer and Operations Research, vol. 27, pp.
935-962, 2000.

[10] K Park, "Fuzzy-set theoretic interpretation of economic order quantity", IEEE Transactions on Systems, Man, and Cybernetics SMC-17,pp. 1082-1084, 1987.

[11] Kweimei Wu, Jing S Yao, "Fuzzy inventory with backorder for fuzzy order quantity and fuzzy shortage quantity", European Journal of Operational Research, vol. 150, no. 2, pp. 320-352, 2003

[12] Lotfi A. Zadeh, "Fuzzy sets," Information and Control, vol. 8, no. 3, pp. 338-353, 1965.

[13] M Ameli, A Mirzazadeh and M A Shirazi, "Economic order quantity model with imperfect items under fuzzy inflationary conditions", Trends in Applied Sciences Research, vol. 6, no.3, pp. 294-303, 2011.

[14] Mitsuo Gen, Yasuhiro Tsujimura, Dazhong Zheng, "An application of fuzzy set theory to inventory control models", Computers and Industrial Engineering, vol. 33, pp. 553-556, 1997.

[15] Mirko Vujosevic, Dobrila Petrovic, Radivoj Petrovic, "EOQ Formula when inventory cost is fuzzy" International Journal Production Economics, vol. 45, pp. 499-504, 1996.

[16] Nagoor A Gani, S. Maheswari, "Economic order quantity for items with imperfect quality where shortages are backordered in fuzzy environment", Advances in Fuzzy Mathematics, vol. 5, no. 2, pp. 91-100, 2010.

[17] Pankaj Dutta, Dabjani Chakraborty, Akhil R Roy, "A single-period inventory model with fuzzy random variable demand", Mathematical and Computer Modeling, vol. 41 , no.8-9, pp. 915-922, 2005.

[18] P M Pu and Y M Liu, "Fuzzy Topology 1, neighborhood structure of a fuzzy point and Moore- Smith Convergence", Journal of Mathematical Analysis and Application, vol. 76, pp. 571-599, 1980.

[19] R Uthaykumar, M Valliathal, "Fuzzy economic production quantity model for weibull deteriorating items with ramp type of demand", International Journal of Strategic Decision sciences, vol. 2, no. 3, pp. 55-90, 2011.

[20] San C Chang, Jing S Yao, Huey M Lee, "Economic reorder point for fuzzy backorder quantity", European Journal of Operational Research, vol. 109, pp. 183-202, 1998.

[21] Sanchyi Chang, "Fuzzy production inventory for fuzzy product quality with triangular fuzzy number", Fuzzy Set and Systems, vol. 107, pp.37-57, 1999.

[22] Saheli S Nezhad, Shima M Nahavandi, Jamshid Nazemi, "Periodic and continuous inventory models in the presence of fuzzy costs", International Journal of Industrial En gineering Computations, vol. 2, pp. 167-178, 2011.

[23] Yu J Lin, "A periodic review inventory model involving fuzzy expected demand short and fuzzy backorder rate", Computers \& Industrial Engineering, vol. 54, no. 3, pp. 666-676,2008. 